\title{
Kranke Kinder: Wer hat Anspruch auf Auskunft?
}

Christina Kotrba

Rechtsanwältin

\begin{abstract}
Einleitung
Ehetrennung und -scheidung sind heute keine Seltenheit mehr - die «Patchwork-Familien» langsam fast die Regel. So stehen die Ärztinnen und Ärzte immer öfter vor dem Problem, ob und wem sie überhaupt Auskunft über den Gesundheitszustand des Kindes geben dürfen. Erschwerend kommen in diesen Situationen oft die schwierigen familiären Umstände hinzu, indem z.B. der obhutsberechtigte Elternteil dem Arzt verbietet, dem anderen Elternteil Informationen über den Gesundheitszustand des gemeinsamen Kindes zu erteilen. Dieser Artikel soll Ärztinnen und Ärzten einen kurzen, nicht abschliessend zu verstehenden Überblick über die rechtliche Lage in der Schweiz vermitteln, um in solchen Situationen angemessen reagieren zu können.
\end{abstract}

Informations- und Auskunftsrecht der Eltern bei gemeinsamer elterlicher Sorge sowie bei alleiniger elterlicher Sorge eines Elternteils

Haben beide Eltern die elterliche Sorge inne (auch wenn nur ein Elternteil die Obhut des Kindes hat), stützen sich die Rechte und Pflichten der Eltern auf den Inhalt der elterlichen Sorge nach Art. 296 ff.

\section{Ärztinnen und Ärzte stehen immer öfter vor dem \\ Problem, ob und wem sie überhaupt Auskunft über den Gesundheitszustand des Kindes geben dürfen.}

Korrespondenz:

lic. iur. Christina Kotrba

Suhr Würgler Maag Bisang

Rechtsanwälte

Riesbachstrasse 57

Postfach 1071

CH-8034 Zürich

Tel. 0434884141

kotrba[at]zurichlawyers.com
ZGB. Beide Elternteile haben in diesem Fall von Gesetzes wegen die Vertretung des Kindes gegenüber Drittpersonen im Umfang der ihnen zustehenden elterlichen Sorge inne (Art. 304 Abs. 1 ZGB). In solchen Fällen hat also z. B. auch der Vater, dessen Kind bei der Mutter wohnt, ein Auskunftsrecht, denn das Auskunftsrecht hat auch der nicht obhutsberechtigte, aber sorgeberechtigte Elternteil kraft elterlicher Sorge.

Hat nur ein Elternteil die elterliche Sorge inne, richten sich die Rechte des nicht sorgeberechtigten Elternteils nach Art. 275a ZGB. Demnach kann der nicht sorgeberechtigte Elternteil bei Drittpersonen, die an der Betreuung des Kindes beteiligt sind, wie namentlich bei Lehrkräften, Ärztinnen und Ärzten, in gleicher Weise wie der Inhaber der elterlichen Sorge Auskünfte über den Zustand und die

\section{Droit d'information et de renseignement des parents de patients mineurs en cas de séparation et de divorce}

II convient de préciser que le législateur garantit aux deux parents, et ce indépendamment du détenteur de l'autorité parentale (ou de la garde), le droit à l'information et aux renseignements. Les droits et devoirs du parent qui détient l'autorité parentale s'appuient sur les articles 296 ss. du Code civil suisse (CC). Si un parent ne détient pas l'autorité parentale, ses droits se fondent sur l'art. $275 \mathrm{a}$ al. 2 CC. En vertu de cet article, le père ou la mère qui ne détient pas l'autorité parentale peut, tout comme le détenteur de l'autorité parentale, recueillir auprès de tiers qui participent à la prise en charge de l'enfant (notamment auprès de ses enseignants ou de son médecin) des renseignements sur son état et son développement. Les deux parents ont ainsi droit à l'obtention d'informations sur l'état de santé de leur enfant. Le ou la partenaire du parent qui exerce l'autorité parentale ne dispose toutefois pas de ce droit, sauf dans les cas où il/elle doit représenter le détenteur de l'autorité parentale.

Entwicklung des Kindes einholen (Art. 275a Abs. 2 ZGB).

Der Gesetzgeber wollte mit Art. 275a ZGB das Verantwortungsbewusstsein des nicht sorgeberechtigten Elternteils fördern und somit erreichen, dass dieser an der Entwicklung seines Kindes teilhaben kann. Selbst wenn also ein Elternteil die elterliche Sorge nicht innehat, so hat dieser Elternteil direkt (gestützt auf das Gesetz) das Recht, von Drittpersonen, die an der Betreuung des Kindes beteiligt sind, Auskünfte über den Zustand und die Entwicklung des Kindes einzuholen. Das Informations- und Auskunftsrecht des nicht sor- 


\section{Begriffserklärung}

Elterliche Sorge: Die elterliche Sorge (Art. 296 ZGB) umfasst Erziehung, Ausbildung und gesetzliche Vertretung des Kindes (Art. 301-306 ZGB) sowie die Verwaltung seines Vermögens (Art. 318 ff. ZGB). Aufgrund der elterlichen Sorge steht den Eltern die Obhut über das Kind zu. Nach der Trennung bleibt die elterliche Sorge in der Regel bei beiden Elternteilen gemeinsam. Das bedeutet, dass beide Eltern für das Kind vollumfänglich verantwortlich sind. Erst bei der Scheidung wird entschieden, ob die Eltern die gemeinsame elterliche Sorge behalten sollen oder aber ob nur einem Elternteil die elterliche Sorge (d.h. alleinige elterliche Sorge) zugesprochen wird. Bei nicht verheirateten Eltern ist die elterliche Sorge in der Regel bei der Mutter (wobei es Ausnahmen gibt, so z.B. bei einer minderjährigen Mutter oder bei Entzug der elterlichen Sorge). Steht ein minderjähriges Kind nicht unter elterlicher Sorge, so ernennt die Kindesschutzbehörde einen Vormund (Art. 327a ZGB). Dem Vormund stehen die gleichen Rechte wie den Eltern zu (Art. 327c Abs. 1 ZGB).

Der Vollständigkeit halber sei hier darauf hingewiesen, dass per 1. Juli 2014 die gemeinsame elterliche Sorge die Regel wird. Auf das Auskunftsrecht der Eltern hat dies jedoch keinen Einfluss.

Elterliche Obhut: Die Obhut ist ein Teil der elterlichen Sorge und umfasst die tägliche Betreuung und Pflege des Kindes sowie die Befugnis, über den Aufenthaltsort des Kindes zu entscheiden (Art. 301 ZGB sowie Art. 25 ZGB). Während der Ehe üben die Eltern die Obhut gemeinsam aus. Bei einer Trennung müssen die Eltern (oder das Gericht bzw. die Kindesschutzbehörde) über die Obhut ihrer Kinder entscheiden. Es wird somit darüber entschieden, bei wem das Kind mehrheitlich wohnen wird. Allein aufgrund der Obhut ist nicht ersichtlich, wer die elterliche Sorge innehat. Die Obhut kann durch richterlichen Entscheid oder durch Entscheid der Kindesschutzbehörde von der elterlichen Sorge getrennt und einem Elternteil alleine zugesprochen werden.

geberechtigten Elternteiles (d.h. bei alleiniger elterlichen Sorge des anderen Elternteils) stützt sich somit ebenfalls direkt auf das Gesetz (Art. 275a Abs. 2 ZGB).

Zusammenfassend kann festgehalten werden, dass das Informations- und Auskunftsrecht somit beiden Elternteilen (unabhängig von der elterlichen Sorge) zusteht.

\section{Auch ein Vater, dessen Kind bei der Mutter wohnt, hat ein Auskunftsrecht.}

\section{Informations- und Auskunftsrecht des Lebenspartners eines Elternteils}

Das Informations- und Auskunftsrecht der Eltern stützt sich auf das Gesetz. Beim Inhaber der elterlichen Sorge ist Art. 296 ff. ZGB Rechtsgrundlage; beim nicht sorgeberechtigten Elternteil Art. 275a Abs. 2 ZGB. Eine entsprechende gesetzliche Regelung zum Informations- und Auskunftsrecht für Lebenspartner oder Lebenspartnerinnen eines Elternteils gibt es hingegen nicht. Grundsätzlich kommt somit dem Lebenspartner oder der Lebenspartnerin eines Elternteils kein Informations- und Auskunftsrecht zu.
Gemäss Art. 299 ZGB hat jedoch jeder Ehegatte dem andern in der Ausübung der elterlichen Sorge gegenüber dessen Kindern in angemessener Weise beizustehen und ihn zu vertreten, wenn es die Umstände erfordern. Art. 299 ZGB normiert demnach die Pflicht und das Recht zur Beteiligung an der elterlichen Verantwortung, wobei Art. 299 ZGB lediglich von Ehegatten des Inhabers der elterlichen Sorge spricht (alleinige oder gemeinsame elterliche Sorge). Art. 299 ZGB kann jedoch auch auf nichteheliche Partnerinnen/Partner analog angewandt werden (BSK ZGB I Ingeborg Schwenzer, N 1 zu Art. 299; BGE 128 IV 154). Das heisst konkret, dass der Stiefelternteil bzw. die Lebenspartnerin oder der Lebenspartner den leiblichen Elternteil in der Ausübung der elterlichen Sorge vertreten kann. Das gilt vor allem dann, wenn es die Umstände erfordern, was z. B. bei dringender ärztlicher Behandlung der Fall ist. In diesen Fällen muss der Lebenspartnerin bzw. dem Lebenspartner des Inhabers der elterlichen Sorge ein Auskunftsrecht zukommen.

\section{Exkurs: Zur Urteilsfähigkeit minderjähriger Patientinnen und Patienten}

In Bezug auf die Auskunftspflicht des medizinischen Fachpersonals bei minderjährigen Patientinnen und Patienten (d.h. losgelöst von der Frage, wem das Sorgerecht zusteht bzw. wer obhutsberechtigt ist, da wie soeben erwähnt, den Elternteilen unabhängig von der elterlichen Sorge ein Auskunftsrecht zukommt) ist $\mathrm{zu}$ unterscheiden, ob Informationen über urteilsfähige oder über urteilsunfähige Kinder und Jugendliche abgegeben werden.

Gemäss Art. 16 ZGB ist urteilsfähig, wem nicht wegen seines Kindesalters, infolge geistiger Behinderung, psychischer Störung, Rausch oder ähnlicher Zustände die Fähigkeit mangelt, vernunftgemäss zu handeln. In Bezug auf ein Kind oder eine(n) Jugendliche(n) hängt die Urteilsfähigkeit von der individuellen Reife ab. Es ist demnach in jedem Einzelfall zu beurteilen, ob die geistig-psychische Reife der vom Gesetz geforderten Vernunft und Selbstverantwortlichkeit entspricht und im Rahmen der konkreten Entscheidung vorhanden ist (vgl. dazu BGE 90 II 9; 107 II 22; 119 II 4; 122 III 401). Die Urteilsfähigkeit ist demnach unabhängig vom Alter zu bestimmen und orientiert sich an der geistigen Fähigkeiten des Kindes bzw. der/des Jugendlichen. Sie bezieht sich dabei immer auf den konkret zu treffenden Entscheid.

\section{Bei urteilsunfähigen Kindern und Jugendlichen}

Geht es um medizinische Eingriffe oder Therapien für eine urteilsunfähige minderjährige Person, muss die gesetzliche Vertretung (i.d.R. die Eltern) zustimmen, soweit es sich nicht um einen Notfall handelt, in dem die Ärztinnen und Ärzte aufgrund ihrer Berufspflicht selbständig das Nötige zu veranlassen haben. Massgebend ist in den dringlichen Fällen bei 


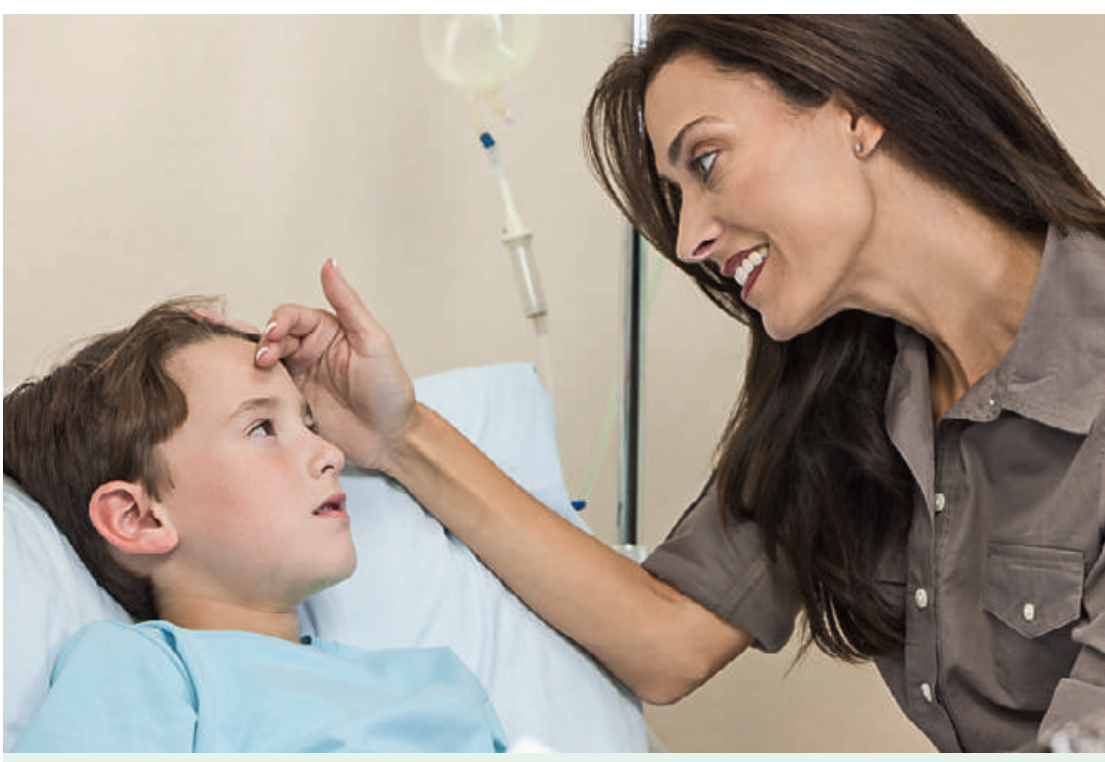

Hat auch der geschiedene Vater ohne Sorgerecht Anspruch auf Informationen über den Gesundheitszustand seines Sohnes?

urteilsunfähigen minderjährigen Personen - wie bei jedem Urteilsunfähigen in einer Notlage - der mutmassliche Wille sowie dessen Interessen (vgl. dazu Art. 379 ZGB).

Die medizinischen Informationen stehen - wie bereits erwähnt - sowohl dem sorgerechtsinnehabenden Elternteil (gestützt auf die elterliche Sorge) wie auch demjenigen Elternteil $\mathrm{zu}$, der das Sorgerecht nicht innehat (gestützt auf Art. 275a Abs. 2 ZGB).

\section{Bei urteilsfähigen Kindern und Jugendlichen}

Ist die handlungsunfähige Person urteilsfähig, so übt sie ihre höchstpersönlichen Rechte selbständig aus (Art. 19c Abs. 2 ZGB) und entscheidet damit auch selbst, welche medizinischen Eingriffe vorgenommen werden sollen und wem die entsprechenden Informationen erteilt werden dürfen (BGE 134 II 235). Ohne Zustimmung der urteilsfähigen minderjährigen Person dürfen Ärztinnen und Ärzte aufgrund Art. 321 StGB (Verletzung des Berufsgeheimnisses) weder der gesetzlichen Vertretung noch dem Elternteil ohne elterliche Sorge Informationen zukommen lassen (Ausnahmen sind vorbehalten: wie z.B. im Falle von Art. 14 und 364 StGB, wenn an der minderjährigen Patientin oder am minderjährigen Patienten eine strafbare Handlung begangen worden ist, oder aber wenn die medizinische Massnahme mit schwerwiegenden finanziellen Konsequenzen verbunden ist, die nicht mehr aus dem freien Kindesvermögen bestritten werden kann [1].
In der Praxis kann dies im Einzelfall zu einer schwierigen Interessenabwägung führen. Als Richtlinie kann festgehalten werden, dass bei Jugendlichen von 12 bis 16 Jahren die Urteilsfähigkeit im Einzelfall beurteilt werden muss; es kommt auf das jeweilige Kind und die Art des Eingriffs an. Ab dem 16. Altersjahr kann von der Urteilsfähigkeit ausgegangen werden, wenn es sich nicht um einen aussergewöhnlichen Eingriff mit erheblicher Tragweite handelt [2]. Bei grösseren Eingriffen mit auch für die Zukunft weitreichenden Auswirkungen (wie z. B. bei einer Organspende) dürfte die Urteilsfähigkeit auch bei einer 17-Jährigen bzw. einem 17-Jährigen nicht in jedem Fall vorliegen. Herrscht Unklarheit über die Urteilsfähigkeit, so muss die Einwilligung sowohl von der minderjährigen Patientin bzw. vom minderjährigen Patienten wie auch vom gesetzlichen Vertreter eingeholt werden [3].

\section{Zusammenfassung}

In Bezug auf das Auskunftsrecht ist im Ergebnis festzuhalten, dass dieses einem Elternteil unabhängig von der elterlichen Sorge (oder der elterlichen Obhut) durch den Gesetzgeber gewährt wird. Beide Elternteile haben Anrecht auf die Information über den Gesundheitszustand ihres Kindes. Die Lebenspartnerinnen oder Lebenspartner des Inhabers der elterlichen Sorge haben grundsätzlich kein Auskunftsrecht, ausser in denjenigen Fällen, in denen die Lebenspartnerin oder der Lebenspartner den Inhaber der elterlichen Sorge vertreten muss.

\section{Literatur}

1 Nägeli M. Die ärztliche Behandlung handlungsunfähiger Patienten aus zivilrechtlicher Sicht. Diss. Zürich 1984; S. 25

2 Schweizerische Akademie der Medizinischen Wissenschaften, Verbindung der Schweizer Ärztinnen und Ärzte FMH (Hrsg.). Rechtliche Grundlagen im medizinischen Alltag, ein Leitfaden für die Praxis. 2. Auflage; 2013. S. 43.

3 In diesem Sinne auch Max Nägeli, a.a. O., S. 104 ff.

\section{Articles interactifs}

Vous souhaitez commenter cet article? II vous suffit pour cela d'utiliser la fonction «Ajouter un commentaire» dans la version en ligne. Vous pouvez également consulter les remarques de vos confrères sous: www.bullmed.ch/ numero-actuel/articles-interactifs/ 